\title{
Développement et évaluation
} d'une activité de formation continue adaptée aux infirmières et infirmiers en traumatologie

\author{
Development and evaluation of a continuing education activity \\ for trauma nurses working in emergency departments
}

\author{
Mélanie MARCEAU, Lise R. TALBOT et Frances GALLAGHER \\ École des sciences infirmières, Faculté de médecine et des sciences de la santé, Université de Sherbrooke, \\ Sherbrooke, Canada
}

Manuscrit soumis le 18 mai 2011 ; commentaires éditoriaux formulés aux auteurs le 26 juillet 2011 ; accepté pour publication le 21 décembre 2012

\author{
Mots-clés \\ Formation continue ; \\ soins infirmiers ; \\ traumatologie ; \\ soins critiques ; \\ besoins de formation \\ continue ; recherche \\ participative
}

\begin{abstract}
Résumé - Contexte : La pratique infirmière en traumatologie est complexe. Pour cette raison, la formation continue (FC) s'avère essentielle au développement et au maintien des compétences des infirmières œuvrant dans ce champ de pratique. But : L'étude visait à développer et évaluer une activité de FC pour les infirmières œuvrant en traumatologie au service des urgences. Sujet/Matériel/Méthode : Un devis qualitatif, inspiré de l'approche de quatrième génération de Guba et Lincoln, a inclus des données qualitatives et quantitatives. L'étude repose sur le cadre conceptuel de Le Boterf. Après avoir identifié les besoins de formation de 30 infirmières du service des urgences d'un centre hospitalier universitaire lors d'entrevues de groupe, une activité de FC a été élaborée. Les connaissances générales et reliées aux procédures ont été évaluées avant, immédiatement après et trois mois après l'activité par le biais de questionnaires et d'entrevues de groupe. Nous avons évalué la satisfaction des participant(e)s à l'aide d'un questionnaire. Des analyses statistiques et qualitatives ont été effectuées. Résultats : L'analyse des besoins a donné lieu à la co-construction d'une activité de FC « sur mesure » pour les infirmières. À la suite de cette formation, les connaissances générales et reliées aux procédures, concernant les soins donnés aux personnes polytraumatisées se sont améliorées et se sont maintenues jusqu'à trois mois après la formation. L'élaboration d'une activité de FC « sur mesure », selon les besoins exprimés, constituait une nouveauté dans le domaine des soins infirmiers en traumatologie et a favorisé la participation. Conclusion : L'approche participative novatrice utilisée dans cette étude a contribué au développement des connaissances, à la mobilisation des ressources personnelles ainsi qu'au sentiment d'implication vécu par le personnel infirmier tout au long du processus de construction et de mise en œuvre des activités de FC.
\end{abstract}




\begin{tabular}{|c|c|}
\hline $\begin{array}{l}\text { Keywords } \\
\text { Continuing } \\
\text { education; trauma } \\
\text { nursing; critical care; } \\
\text { continuing education } \\
\text { needs; participatory } \\
\text { research }\end{array}$ & $\begin{array}{l}\text { Abstract - Context: Since trauma nursing is complex, continuing education (CE) is essential } \\
\text { to the development and maintenance of the skills of trauma nurses. Purpose: The purpose of } \\
\text { the study was to develop and evaluate a CE activity for trauma nurses working in emergency } \\
\text { departments. Subject/Materials/Methods: A 4th generation evaluative design incorporating } \\
\text { qualitative and quantitative data was used. The study was based on the conceptual framework } \\
\text { of Le Boterf. After identifying the training needs of } 30 \text { emergency department nurses during } \\
\text { group interviews, a CE activity was developed. General and procedural knowledge was eval- } \\
\text { uated by questionnaires and group interviews before and after the activity and three months } \\
\text { later. A questionnaire was also used to evaluate the satisfaction of the participants. Statistical } \\
\text { and qualitative analyses were performed. Results: The needs analysis led to the development } \\
\text { of a customized CE activity for trauma nurses. General and procedural knowledge of the care } \\
\text { given to polytrauma victims improved after the training and the improvement was maintained } \\
\text { three months later. The customized approach and use of active teaching strategies fostered } \\
\text { participation. Conclusion: The innovative participatory approach used in this study contrib- } \\
\text { uted to the nurses' feeling of being involved throughout the process of developing and imple- } \\
\text { menting a CE activity. }\end{array}$ \\
\hline
\end{tabular}

\section{Introduction}

Les personnes polytraumatisées représentent une proportion importante des patients admis au service des urgences. Dans ce milieu de soins spécialisés, l'infirmier(ère) doit gérer une gamme de situations cliniques complexes dont l'évolution demeure imprévisible. Pour agir avec compétence, ces professionnels doivent intervenir en se fondant sur des normes de pratique et des résultats probants ${ }^{[1]}$. La formation continue (FC) est dès lors un moyen indispensable pour favoriser l'actualisation des connaissances, le maintien des standards de pratique ainsi que le développement des compétences ${ }^{[2-5]}$.

La FC se définit comme étant un processus d'apprentissage continu, planifié pour améliorer les connaissances, les attitudes et les habiletés des infirmiers(ères) et elle est mise en œuvre, notamment, à partir des besoins exprimés ${ }^{[3]}$. La FC en traumatologie peut contribuer à l'amélioration de la prestation des soins tout en augmentant la rapidité et la qualité des interventions ${ }^{[6]}$. De plus, des études démontrent que la FC diminue la mortalité ainsi que la morbidité et facilite la réadaptation chez les personnes soignées ${ }^{[7,8]}$.

Selon une revue systématique de la littérature, la FC résulte rarement d'une évaluation des besoins des infirmiers(ères). Des écrits en pédagogie, en médecine et en sciences infirmières relèvent que certaines méthodes d'enseignement sont efficaces en FC, principalement celles qui recourent à une approche participative $e^{[3,7,14]}$. La recension des écrits et nos expériences cliniques indiquent que la FC en traumatologie s'avère essentielle ${ }^{[5,6]}$, que les collègues de la discipline la réclament et qu'elle doit reposer sur les besoins exprimés ${ }^{[3,4,15]}$.

La présente étude avait pour but de développer et d'évaluer une activité de FC pour les infirmiers(ères) du service des urgences travaillant en traumatologie. Les objectifs spécifiques de cette étude visaient à : 1) identifier des besoins spécifiques de FC en traumatologie exprimés par des infirmiers(ères) du service des urgences ; 2) évaluer leurs connaissances générales et procédurales avant, immédiatement après et trois mois après une activité de FC et enfin : 3 ) évaluer la satisfaction des participant(e)s concernant la réponse à leurs besoins de formation.

Cette recherche se distingue des études rapportées dans la littérature par le fait qu'elle concerne une activité de formation élaborée et mise en œuvre à partir d'une évaluation des besoins d'apprentissage des infirmiers(ères).

\section{Cadre conceptuel}

Cette étude s'inspire du cadre conceptuel qui rend compte de la notion de compétence, tel que l'a développé Le Boterf ${ }^{[16]}$. La figure 1 regroupe l'ensemble des composantes du «savoir agir avec 


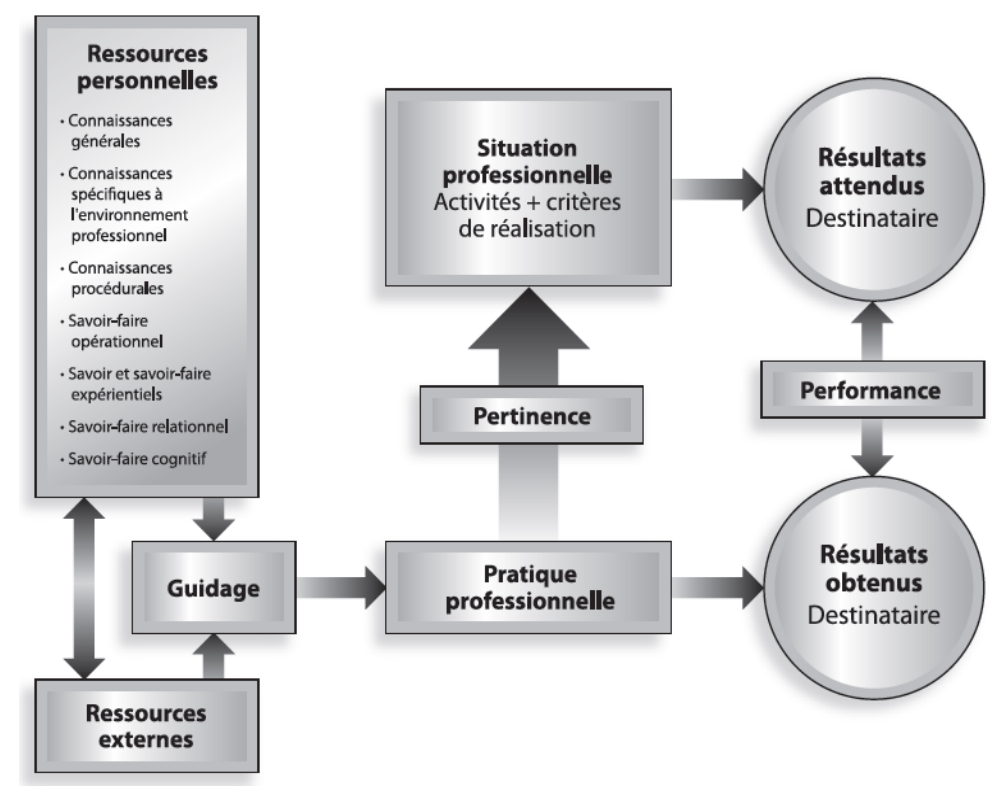

Fig. 1. Savoir agir avec compétence (d'après Le Boterf ${ }^{[16]}$ ).

compétence » ${ }^{[16]}$. Selon ce modèle, dans une situation professionnelle, soit une situation clinique, l'infirmier(ère) compétent(e) combinera et mobilisera les ressources personnelles et externes nécessaires à la réalisation d'une activité complexe de soins ${ }^{[16]}$. Les ressources personnelles rassemblent les connaissances générales, les connaissances spécifiques sur l'environnement professionnel, les connaissances procédurales, le savoir-faire opérationnel, les savoirs et savoir-faire expérientiels, le savoir-faire relationnel ainsi que le savoir-faire cognitif, qui peuvent être développés grâce à la FC. Les ressources externes, que sont par exemple les ouvrages de références ou l'expertise de certains collègues, proviennent de l'environnement clinique. Le choix des composantes découle de différents types de guidage, tels que le guidage émotionnel (émotions), le guidage cognitif (raisonnement clinique), le guidage selon les règles professionnelles (règles de l'art) et le guidage éthique (valeurs). Le Boterf ${ }^{[16]}$ définit le guidage comme une action qui permettra au professionnel « de choisir et de conduire des pratiques pertinentes et de combiner et mobiliser des ensembles de ressources qui lui sont nécessaires » ${ }^{[16]}$ (p. 82).
La situation professionnelle, telle qu'une situation clinique, se compose d'une activité prescrite à réaliser, d'un ensemble de critères de réalisation souhaitables ainsi que des résultats attendus au service d'un destinataire. La pratique professionnelle se définit plutôt comme une activité clinique réelle et une façon d'agir ayant pour conclusion des résultats réels qui sollicitent une combinaison de ressources disponibles. On observe que le milieu réel (pratique professionnelle) dévie parfois des standards de pratique (situation professionnelle), qui définissent les exigences de la profession infirmière.

Dans le cadre de cette étude, l'évaluation des besoins de FC des infirmiers(ères) portait sur leurs ressources personnelles en termes de connaissances générales et procédurales. Ces dernières font partie des connaissances d'action, qui se distinguent des connaissances déclaratives selon la typologie formelle de la psychologiecognitive ${ }^{[17]}$,repriseparTardif ${ }^{[18]}$.L'évaluation portait aussi sur les ressources externes, plus précisément les attentes des infirmiers(ères) concernant les composantes pédagogiques de l'activité de FC (ex. : méthodes pédagogiques, durée et horaire, personne formatrice, matériel pédagogique). 


\section{Matériel et méthodes}

Un dispositif de recherche évaluative, inspiré de l'approche dite de quatrième génération selon Guba et Lincoln ${ }^{[19]}$, incluant des données qualitatives et quantitatives, a été utilisé. Dans le cadre d'une telle approche, le milieu professionnel est associé à l'identification de la problématique, ce qui implique la collaboration des infirmiers(ères) concerné(e)s par la situation. Des nuances sont appliquées à la notion d'objectivité puisque les chercheurs interagissent avec les participant(e)s et tiennent compte de la perception de tous. La notion de collaboration implique que les gens concernés par la situation co-construisent l'activité de formation continue avec les chercheurs. De plus, ce dispositif de recherche repose sur un processus de négociation entre des personnes parties prenantes (stakeholders) de l'évaluation, à savoir : a) les infirmiers(ères) du service des urgences qui ont accepté de participer à l'étude ; b) le comité de direction, formé de l'infirmière-chef du service des urgences et des trois auteurs de l'article, qui a collaboré à l'ensemble du processus de recherche et : c) le comité élargi, composé de représentants du syndicat, de la direction des ressources humaines ainsi que de l'équipe médicale, qui a aidé à pallier les contraintes potentielles du milieu.

\section{Participantes et participants}

Initialement, le protocole de recherche prévoyait que l'échantillon devait comporter dix participant(e)s. Les critères de sélection pour participer à l'étude se résumaient ainsi: a) être infirmier(ère) depuis au moins six mois afin de mieux cerner ses besoins de $\mathrm{FC}$; b) être orienté au service des urgences depuis au moins six mois et c) être titulaire du poste ou effectuer un remplacement à long terme. Pour effectuer le recrutement, des feuillets informatifs et des rencontres avec les infirmiers(ères), avant ou après leur quart de travail, ont permis de leur expliquer la recherche et de répondre à leurs questions. Au total, nous avons rencontré 65 personnes, infirmiers(ères) du service des urgences, parmi 74 possibles $(88 \%)$. Les personnes intéressées pour participer à la recherche remplissaient un coupon-réponse dans un délai de deux semaines suivant la séance d'information. Elles le déposaient dans une boîte scellée prévue à cette fin, laquelle se trouvait dans la salle de pause du service des urgences.

\section{Collecte de données}

La collecte des données comportait trois étapes distinctes. Dans un premier temps (T1), les infirmiers(ères) remplissaient un questionnaire de renseignements sociodémographiques et participaient à une entrevue de groupe focalisée (focus group) visant à explorer leurs besoins de FC en traumatologie, leur conception d'une formation idéale, les modalités d'enseignement favorisées ainsi que les facteurs optimisant et contraignant leur participation à des activités de FC. Compte tenu du nombre élevé de participant(e)s au T1 $(n=30)$, ils ont été divisés en trois sous-groupes pour favoriser les échanges lors de la première entrevue de groupe focalisée. Les résultats du premier groupe ont été partagés avec le deuxième groupe puis les résultats des premier et deuxième groupes ont été transmis au troisième groupe. Cette validation en boucle a servi à établir un consensus final auprès des trois groupes concernant les thèmes de formation prioritaires, les modalités pédagogiques préférées et le moment favorable à la tenue de l'activité de formation. Par la suite, ces thèmes ont fait l'objet d'un échange auprès des parties prenantes, ce qui a conduit à la construction de l'activité de FC.

Dans un deuxième temps (T2), lors de la journée de l'activité de FC, les participant(e)s répondaient à un questionnaire d'évaluation des connaissances générales et procédurales pré et post formation. Le questionnaire, construit aux fins de cette recherche, portait sur les éléments du Trauma Nursing Core Course (TNCC) ${ }^{[20]}$ ayant fait l'objet de l'activité de FC. Il comportait 20 questions à réponses ouvertes et courtes ou à choix multiples. Chaque bonne réponse valait un point, pour un score total de 20 points. Des experts (trois infirmières spécialisées en soins critiques) ont validé le questionnaire en examinant la pertinence et la clarté de chacune des questions, ce qui assurait une meilleure validité de contenu ainsi qu'une meilleure validité apparente ${ }^{[21]}$. Le questionnaire a également été prétesté auprès de deux infirmiers 
volontaires de service des urgences, ce qui a permis d'évaluer, entre autres, la durée et le niveau de difficulté du questionnaire.

Parmi les questions, 11 évaluaient les procédures (examen physique, dégagement des voies respiratoires) et neuf autres les connaissances générales (évaluation initiale et secondaire, choc, pédiatrie). Le temps nécessaire pour répondre au questionnaire était approximativement de 30 minutes. À la toute fin de la journée de FC, un questionnaire avec une échelle de type Likert à quatre niveaux de réponses (tout à fait satisfait, plutôt satisfait, plutôt insatisfait et insatisfait) permettait de documenter la satisfaction des participant(e)s en regard de l'organisation de la formation, des personnes formatrices, des parties théorique et pratique, de même que leur appréciation générale de l'ensemble de l'activité de FC.

Enfin, les infirmiers(ères) se rencontraient une troisième fois (T3), trois mois après avoir participé à l'activité de FC, pour une deuxième entrevue de groupe focalisée afin de répondre de nouveau au questionnaire sur les connaissances et exprimer leur appréciation en regard de la formation. Lors de cette entrevue, une discussion invitait les infirmiers(ères) à décrire comment ils (elles) intégraient leurs nouveaux apprentissages dans leur pratique professionnelle ${ }^{[16]}$. De façon plus explicite, les participant(e)s présents racontaient une situation clinique où ils avaient mobilisé des ressources personnelles acquises lors de l'activité de FC, ainsi qu'une situation clinique où ils avaient eu des difficultés à appliquer les conduites proposées.

Les deux entrevues de groupes focalisées, d'une durée de deux heures, ont été conduites à l'aide de guides semi-structurés conçus à cet effet et construits de façon rigoureuse selon les étapes suggérées par Morgan et Kruger ${ }^{[22]}$. Ils ont été validés par les deux directrices. Les guides se composaient de thèmes précis à explorer, tout en rendant possible l' ajout de questions visant à approfondir les réponses des personnes interrogées. Le choix des thèmes retenus découlait de la recension des écrits sur la FC et la traumatologie. Comme il s'agissait d'obtenir un consensus, l'entrevue de groupe s'avérait plus appropriée que l'entrevue individuelle. Le nombre idéal de participant(e)s se situe entre 6 et 12 personnes $^{[22]}$ mais des limites organisationnelles ont obligé les chercheurs à créer de plus grands groupes.

\section{Analyse des données}

Les données qualitatives provenant des entrevues de groupe ont été enregistrées, transcrites et analysées selon une méthode inspirée des travaux de Huberman et Miles ${ }^{[23]}$. La grille d'analyse, élaborée par les trois auteurs, comportait des catégories prédéterminées découlant de la recension des écrits auxquelles se sont ajoutées des catégories émergentes. Les catégories composant la grille concernaient les besoins prioritaires de FC en termes de connaissances générales et procédurales, les modalités d'apprentissage préférées, les facteurs contraignant et les facteurs favorisant la participation à des activités de $\mathrm{FC}$ et la mobilisation dans leur pratique professionnelle des connaissances générales et procédurales acquises lors de la FC.

Par la suite, une analyse thématique plus circonscrite a permis de raffiner la grille de classification afin de répondre le plus précisément possible aux objectifs de recherche. Des catégories émergentes ont été ajoutées en cours d'analyse pour condenser l'ensemble des données. L'étudiante et ses deux directrices ont analysé individuellement le contenu des entrevues de groupe afin de permettre une compréhension commune de l'ensemble du matériel et d'obtenir par la suite un consensus inter-investigateurs.

Nous avons effectué des analyses statistiques descriptives sur les données sociodémographiques pour établir le profil des participant(e)s. Les réponses aux questionnaires d'évaluation des connaissances, respectivement administrés en pré et post $\mathrm{FC}$, ont fait l'objet d'analyses descriptives et corrélationnelles. Étant donné l'absence d'une distribution normale des résultats, le test des rangs de Wilcoxon a permis d'effectuer les comparaisons entre les différents temps de mesure. Le seuil de signification a été fixé à 0,05 et les analyses ont été effectuées à l'aide du logiciel SPSS version 17.0.

Les personnes participantes ont été informées des objectifs de la recherche, de la nature de leur participation puis ont signé le formulaire de consentement. La participation volontaire permettait à toute 
personne participante de mettre fin à son implication à tout moment et ce, sans préjudice. Les chercheurs ont effectué l'étude dans le respect de l'anonymat et de la confidentialité à tout moment, mesure d'autant plus importante que l'étudiante provenait du milieu clinique où se déroulait la recherche. Une clé, connue seulement par les directrices de maitrise, a été utilisée lors de la codification des données nominales afin de préserver l'anonymat. Une des deux directrices a entreposé sécuritairement les données audionumériques et papier dans son bureau. Ces données doublées conservées pour une durée déterminée de cinq ans, seront détruites par la suite.

\section{Résultats}

\section{Caractéristiques des personnes participantes}

La taille de l'échantillon a été revue à la hausse en raison de l'intérêt marqué des infirmiers(ères) pour la FC en traumatologie. Le nombre de participant(e)s a toutefois fluctué tout au long de la recherche en raison de leurs occupations personnelles ou encore de l'impossibilité de libérer le personnel dans un contexte de pénurie. En effet, 30 infirmier(ères) ont participé au premier groupe focalisé, 27 ont assisté à l'activité de FC puis 15 membres du personnel ont maintenu leur participation jusqu'à la fin.

La moitié des infirmiers(ères) était âgée de 25 à 34 ans et les femmes représentaient $67 \%$ de l'échantillon. Les deux tiers (64\%) des personnes participantes n'avaient pas d'enfant. Le nombre d'années d'expérience professionnelle des personnes de l'échantillon variait de moins de trois ans à plus de 10 années. La majorité des participant(e)s travaillait durant le quart de soir ou de nuit. Par ailleurs, il est important de noter que $76 \%$ des participant(e)s n'avaient jamais reçu de FC en traumatologie avant cette recherche.

Lors de la première entrevue de groupe focalisée, les participant(e)s ont mentionné qu'ils s'impliquaient dans la recherche afin de développer de nouvelles connaissances ainsi que leur confiance en leur capacité d'intervenir auprès d'un patient polytraumatisé. Pour les infirmiers(ères) nouvellement diplômés et moins expérimentés, la FC représentait un moyen d'uniformiser leur pratique : «C'est un projet qui peut nous amener plus de connaissances, puisqu'ils nous consultent, nous les gens sur le plancher pour savoir qu'est-ce qu'on veut exactement dans notre milieu de travail $[\ldots] »$.

\section{Conception de l'activité de formation continue selon les besoins exprimés}

Unanimement, les participant(e)s souhaitaient une FC portant sur des sujets intéressants, comportant des applications pratiques et s'adaptant au contexte du milieu de travail. De plus, elle devait être accessible, dynamique, réaliste et donnée par des personnes formatrices possédant une expertise reconnue. Enfin, la rémunération et la libération du personnel représentaient un incitatif à la participation à de telles activités. Les participant(e)s ont identifié ce qui constituait des barrières à leur participation à une activité de FC, à savoir: les contraintes familiales, les problèmes d'accessibilité, les coûts parfois élevés des activités de $\mathrm{FC}$, le recours à des méthodes pédagogiques axées sur la présentation de contenus théoriques ou une formation sur un thème. Ils percevaient tout particulièrement que, si la formation avait été offerte en dehors des heures de travail, la difficulté à libérer le personnel, dans le contexte actuel de pénurie pourrait aussi réduire l'accès aux activités de FC.

Parmi tous les besoins exprimés, les participant(e)s aux trois groupes de discussion ont souligné l'acquisition de connaissances générales sur les phénomènes du choc et du traumatisme pédiatrique. En ce qui concerne les connaissances procédurales, les besoins prioritaires répertoriés portaient sur l'évaluation initiale et secondaire, le dégagement des voies respiratoires et l'examen physique. Une formation interactive, en petit groupe, s'échelonnant dans le temps et intégrant une variété de méthodes pédagogiques, représentait un atout selon les infirmiers(ères). L'analyse de situations simulées, l'utilisation de vidéos et de cas vécus, la discussion de cas, la lecture ou des exposés magistraux sont des exemples de méthodes pédagogiques énumérées par les personnes participantes. Un infirmier souligne les 
Tableau I. L'activité de formation continue.

\begin{tabular}{lll}
\hline & Partie théorique & Partie pratique \\
\hline Durée & 3 heures & 3 heures \\
Formateur & Duo de formateurs infirmière-médecin & \\
Thèmes abordés de l'activité de & Évaluation initiale et secondaire & Examen physique \\
formation continue & Choc & Dégagement des voies respiratoires \\
& Pédiatrie & Manipulation et mises en situation \\
Méthodes pédagogiques & Présentation magistrale & au laboratoire d'anatomo-pathologie \\
& Démonstrations sur mannequin & Matériel nécessaire aux ateliers \\
Ressources & Remise d'un cahier de référence & Mannequins (enfant et bébé) \\
& Document PowerPoint utilisé au & Manteres \\
\hline
\end{tabular}

Tableau II. L'évolution des connaissances entre les trois temps de mesure.

\begin{tabular}{lccc}
\multicolumn{1}{c}{ Thèmes } & $\begin{array}{c}\text { T1 } \\
\text { Médiane (EIQ) }\end{array}$ & $\begin{array}{c}\text { T2 } \\
\text { Médiane (EIQ) }\end{array}$ & $\begin{array}{c}\text { T3 } \\
\text { Médiane (EIQ) }\end{array}$ \\
\hline $\begin{array}{l}\text { Score total des connaissances } \\
\text { sur 20) }\end{array}$ & $11,2(9,6-12,0)$ & $15,6(13,3-16,4)^{\dagger}$ & $15,4(14,8-16,0)^{\dagger}$ \\
$\begin{array}{l}\text { Connaissances générales } \\
\text { sur 20) }\end{array}$ & $10,9(7,8-13,3)$ & $16,0(14,1-18,7)^{\dagger}$ & $17,1(15,3-18,7)^{\dagger}$ \\
$\begin{array}{l}\text { Connaissances relatives } \\
\text { aux procédures (sur 20) }\end{array}$ & $11,2(9,7-12,2)$ & $14,6(14,0-15,8)^{\dagger}$ & $13,8(13,1-15,5)^{\dagger}$ \\
\hline
\end{tabular}

${ }^{\dagger} p<0,005,{ }^{\ddagger} p<0,001$

EIQ : étendue interquartile $\left(25^{\mathrm{e}}-75^{\mathrm{e}}\right.$ percentile $)$

$\mathrm{T} 1$ : avant l'activité de formation ; T2 : immédiatement après l'activité de formation ;

$\mathrm{T} 3$ : trois mois après l'activité de formation.

avantages des ateliers pratiques ou des laboratoires : "C'est plus ça. Du terre à terre, du pratico-pratique. [...] On a des connaissances, on peut les mettre en application ».

Les participant(e)s ont suggéré plusieurs options d'horaire pour l'activité de FC, avec ou sans libération de personnel. La préférence s'orientait vers une activité de FC sous forme de demi-journées ou de journées intensives se déroulant en semaine et préférablement sans avoir à travailler dans l'unité avant ou après l'activité. En plus des facteurs favorisant leur participation, les participant(e)s ont proposé qu'un duo infirmière-médecin soit responsable de l'activité de FC. Des personnes ressources potentielles ont été nommées en raison de leur expertise reconnue par le milieu.

L'activité de formation a été construite de manière consensuelle à partir des besoins des participant(e)s, avec la collaboration des personnes formatrices et des différentes parties prenantes. Le contenu était basé sur le TNCC ${ }^{[20]}$ (tableau I). Ainsi, après une validation, entres autres, auprès du personnel infirmier, une activité de FC d'une durée de six heures a été offerte à deux groupes de participant(e)s ( $n=12$ et $n=15$ ) ; elle comprenait une partie théorique en matinée et une partie pratique en après-midi. Comme toutes les personnes n'apprennent pas de la même manière $^{[18]}$, différentes activités d'enseignement et d'apprentissage ont été utilisées, telles que l'exposé interactif $^{[18]}$, la démonstration et la simulation ${ }^{[9]}$.

\section{Acquisition de connaissances}

Le tableau II montre que les connaissances générales, les connaissances relatives aux procédures et le score total (maximum 20) obtenu au questionnaire de connaissances se sont améliorés de façon significative immédiatement après l'activité (T2) $(p<0,001)$. 
Toutefois, aucune différence significative n'aététrouvée entre les scores obtenus respectivement après l'activité de FC (T2) et trois mois plus tard (T3), mais les connaissances se sont maintenues dans le temps. Afin de mieux comparer les connaissances relatives aux procédures et les connaissances générales avec le score total, les résultats de ces deux types de connaissances ont été pondérés sur 20.

La deuxième entrevue de groupe focalisée permettait de verbaliser relativement à la mobilisation des connaissances acquises pendant la FC dans la pratique professionnelle. Les participant(e)s ont eu le sentiment d'être mieux outillés et mieux préparés à intervenir auprès de la population polytraumatisée. Ils ont exprimé plus d' assurance dans l'accomplissement de leur travail, plus de pouvoir décisionnel ainsi qu'une diminution de l'anxiété et du stress : "Avec ce qu'on a vu dans la journée de formation, ça m'a donné plus d'assurance [...]. Je vais être plus à l'aise [...]. Je vais prendre plus ma position dans la salle, j'aurai plus de leardership».

Les personnes participantes ont dit être en mesure de mieux réagir aux besoins des patients et d'intervenir avec plus de rapidité. L'équipe infirmière faisait aussi preuve d'un meilleur sens de l'organisation et de plus d'assurance grâce, entre autres, à une compréhension plus précise du rôle des autres intervenantes et intervenants, tels que celui du médecin ou de l'inhalothérapeute. Une amélioration des habiletés au plan de l'évaluation et de la collaboration professionnelle a été rapportée : "[...] je suis plus capable d'évaluer le patient, d'être précis, de prendre mon temps, d'être capable d'aller voir après le médecin et d'expliquer ce que c'est». Cependant, certains facteurs contextuels tels que la pénurie en personnel infirmier ou la surcharge de travail ont entravé la mobilisation adéquate des connaissances générales et procédurales : "On ne peut pas rien appliquer de ce qu'on a appris dans ce temps-là [...]».

\section{Satisfaction face à l'activité de formation continue}

Du point de vue de tous les participant(e)s, l'activité de FC a été une réussite en regard de l'approche participative, de la réponse à leurs besoins tant au plan organisationnel qu' au plan des contenus théoriques et pratiques, et du document de référence : "Ce que j'aime, c'est qu'on a un document qui nous reste, auquel on peut référer ». D'ailleurs, sur l'échelle de Likert du questionnaire de satisfaction, la majorité des participant(e)s se sont dits très satisfaits de la FC. Les participant(e)s ont manifesté le souhait que leur implication dans la recherche incite davantage le milieu de travail à offrir régulièrement de la formation et à libérer le personnel, dans l'optique de susciter la participation à d'autres activités: "Et la formation, on n'en ajamais assez. Et en même temps, ça va stimuler peutêtre la direction en voyant que tous les gens se mobilisent pour un projet de recherche [...] Que, oui, les gens sont intéressés à avoir de la formation puis, oui, on en veut ».

Pour les FC ultérieures, les infirmiers(ères) apprécieraient de courtes activités, variées, interactives et plus fréquentes. Ils ont verbalisé à maintes reprises l'importance d'intégrer une partie pratique dans les activités de FC et ce, par le biais de petits groupes afin de favoriser la participation des gens. Ainsi, les infirmiers(ères) pourraient pratiquer à tour de rôle et développer leurs connaissances générales et procédurales avec le soutien des formateurs et de leurs pairs. Une autre suggestion portait sur l'adaptation des activités de FC aux différents niveaux d'expertise du personnel infirmier.

\section{Discussion}

L'étude rapportée explorait l'intérêt d'une activité de FC réalisée pour et par les personnes concernées, en réponse aux besoins de formation en traumatologie des infirmiers(ères) œuvrant au service des urgences. L'hypothèse générale était qu'une approche participative favorisait l'utilisation des ressources en place, optimisant la faisabilité de la recherche ainsi que le transfert et l'intégration immédiate des connaissances $^{[24]}$. La participation de ces professionnels de la santé à l'activité de FC témoigne du potentiel de cette approche pour répondre à leurs besoins de formation, ce qui constitue le premier constat que nous établissons. Les autres constats concernent les retombées en termes de mobilisation des ressources 
personnelles acquises durant la formation et les facteurs influençant la participation à la FC.

\section{Réponse aux besoins de formation continue}

Plusieurs indices accréditent l'idée que l'activité de FC a répondu aux besoins de FC en traumatologie exprimés par les personnes participant à cette étude. En effet, les propos échangés lors de la rencontre de groupe trois mois après la formation, de même que l'évaluation des connaissances corroborent cette observation. Ainsi, la participation à l'activité est associée à une augmentation des connaissances et à une mobilisation des ressources acquises dans la pratique clinique par les infirmiers(ères).

D'ailleurs, le niveau de connaissances s'est maintenu dans le temps, contrairement à ce qui a été observé dans une autre étude sur une formation continue en traumatologie ${ }^{[8]}$. Les résultats de la présente étude mettent en évidence une augmentation des connaissances entre le début de la journée de formation (T1) et la fin de la journée de formation (T2) et montrent que le niveau de connaissance s'est globalement maintenu jusqu'à trois mois après la formation (T3). Il est possible que le maintien des connaissances dans le temps ait été favorisé par une formation construite sur mesure et adaptée aux besoins des infirmiers(ères). Les personnes participantes provenant d'un même milieu clinique pouvaient se soutenir entre elles pour consolider leurs connaissances et mobiliser les ressources nécessaires à la mise en œuvre de leur compétence à intervenir auprès des personnes polytraumatisées, ce qui n'était pas le cas dans l'étude de Tippett $^{[8]}$.

Les connaissances générales et procédurales se sont maintenues trois mois après l'activité de FC. L'effet plafond entre les T2 et T3 peut s'expliquer par l'absence de rétroaction ou de rafraichissement après la journée de FC. En effet, dans une pédagogie centrée sur l'apprenant, la rétroaction favorise davantage l'acquisition et le transfert des nouvelles connaissances $^{[25,26]}$. Une formation étalée dans le temps avec des activités de formation supplémentaires ainsi qu'un retour sur leurs réponses aux questionnaires d'évaluation des connaissances auraient pu aider les participant(e)s à améliorer leurs difficultés et à faciliter l'intégration des connaissances ${ }^{[10]}$. Finalement, la formule utilisée correspondait à plusieurs égards aux attentes des personnes participantes. Parmi les points forts de l'activité de FC, soulignons son caractère sur mesure, c'est-à-dire fondé sur une évaluation des besoins, ses méthodes pédagogiques dynamiques et interactives. Ainsi, la conception et la réalisation de l'activité reposaient sur des principes pédagogiques reconnus $^{[9,15,24]}$, ce qui peut expliquer les résultats obtenus.

\section{Perception de la mobilisation des ressources personnelles au décours de la formation continue}

Par ailleurs, cette formation a été un moyen efficace pour soutenir le développement des connaissances générales et procédurales des infirmiers(ères) dans un contexte de pénurie et de la novicité de l'équipe infirmière. La FC du personnel infirmier est essentielle à la qualité des soins de la clientèle polytraumatisée, qui requiert des connaissances et des habiletés complexes qui sont en constante évolution ${ }^{[7,8,14]}$.

Concernant la mobilisation des ressources dans leur pratique professionnelle à la suite de l' activité de FC, les participant(e)s ont perçu une augmentation de la confiance en soi, une exécution d'interventions de meilleure qualité, une application des standards de pratique et une plus grande rapidité d'intervention. Ces bénéfices sont d'ailleurs bien documentés dans la littérature ${ }^{[6]}$. Selon Le Boterf ${ }^{[16]}$, les résultats obtenus dans une situation clinique divergent parfois des résultats attendus, en raison de la difficulté rencontrée dans la mobilisation des ressources dans les situations plus complexes de soins. Les participant(e)s ont d'ailleurs mentionné cet écart, surtout dans les situations complexes de soins. Par conséquent, les participant(e)s auraient bénéficié d'un soutien à plus long terme. L'utilisation d'un portfolio ou la fourniture d'une rétroaction à la suite de l' activité de FC auraient permis de mieux documenter les situations cliniques où des ressources ont été mobilisées et d'objectiver le progrès des apprentissages ${ }^{[25,26]}$. 
Facteurs influençant la participation

L'implication des personnes participantes et l'augmentation de leurs connaissances ${ }^{[9]}$ ont été influencées par l'approche participative utilisée, inspirée de Guba et Lincoln ${ }^{[19]}$. La crédibilité des acteurs impliqués dans la FC ainsi que les modalités pédagogiques choisies ont contribué au succès de la formation. D'ailleurs, la suggestion des participant(e)s d'avoir recours à des collègues comme formateurs rejoint l'idée d'Armour et Yelling ${ }^{[24]}$, selon laquelle les apprenants tiennent en plus haute estime l'expertise de leurs collègues que celle des experts extérieurs. Reconnus pour favoriser la participation, des facteurs intrinsèques au milieu tels que le soutien et la reconnaissance des employeurs, de même que le sens des responsabilités professionnelles des infirmier(ères) peuvent aussi expliquer les résultats ${ }^{[27]}$.

Tel qu'identifié par les infirmiers(ères) de la présente étude, les contraintes familiales de même qu'une activité de FC peu accessible, longue et coûteuse freinent la participation, comme l'ont soulevé d'autres auteurs ${ }^{[2,3,27]}$. Malheureusement, le caractère ponctuel de l'activité de $\mathrm{FC}$, le financement limité ainsi que le nombre élevé de personnes participantes ont obligé les chercheurs à s'adapter à la réalité du milieu clinique. Ceci a eu pour conséquence d'offrir l'activité de FC à de plus grands groupes de formation. Or, les activités de FC offertes à des groupes de taille restreinte sont préférables à celles s'adressant à des groupes plus nombreux ${ }^{[28,29]}$, En effet, pour favoriser le caractère interactif de la formation et la participation de chacun ${ }^{[28,29]}$, la taille idéale des groupes est souvent estimée entre sept et 10 personnes, alors que dans notre étude, les sous-groupes étaient formés de 12 et de 15 personnes.

Forces et limites de la recherche

Le dispositif de recherche inspiré de l'évaluation de $4^{\mathrm{e}}$ génération ${ }^{[19]}$ constituait une force de l'étude. Une approche participative a permis de construire une activité de FC sur mesure, basée sur les besoins des infirmiers(ères) du milieu en question, tout en ayant le soutien des gestionnaires (parties prenantes). Cette façon novatrice de concevoir une activité de FC a été appréciée et a eu pour effet de susciter la participation d'un grand nombre d'infirmiers(ères). À première vue, elle peut paraître coûteuse en raison du temps consacré à l'évaluation et à l'obtention d'un consensus avec les personnes concernées. Cependant, ce sont des démarches qui favorisent la mobilisation du milieu dans la mise en œuvre d'activités de formation continue.

Le cadre de référence de Le Boterf ${ }^{[16]}$ a guidé le développement des outils de collecte et d'analyse de données ${ }^{[21]}$. Les connaissances générales et procédurales identifiées et choisies par les participant(e)s lors de la co-construction de l'activité de FC ont été transformée en ressources pouvant être mobilisées par les infirmiers(ères) pour savoir agir avec compétence ${ }^{[16]}$. Le cadre conceptuel ${ }^{[16]}$ n'a pas pu être utilisé intégralement par manque de temps et de ressources. Ainsi, il a été impossible d'évaluer le savoir faire opérationnel, c'est-à-dire l'impact dans le contexte réel de soins, ce qui aurait été une valeur ajoutée de l'étude ${ }^{[16]}$.

L'augmentation des connaissances et la mobilisation des ressources dans la pratique professionnelle pourraient être favorisées par une FC s'échelonnant dans le temps ainsi que par une rétroaction après le questionnaire d'évaluation des connaissances. De plus, une FC davantage contextualisée pourrait possiblement diminuer l'écart entre la réalité du milieu (pratique professionnelle) et les standards de pratique (situation professionnelle) qui définissent les exigences de la profession infirmière.

La crédibilité (validité interne) renvoie « àla justesse et à la pertinence du lien établi entre les données empiriques et leur interprétation ${ }^{[30]}$ (p. 384). Les entrevues de groupe focalisées, enregistrées sur bande audio, ont toutes été transcrites intégralement. Le contenu a été analysé par trois personnes, favorisant ainsi une analyse plus exhaustive et en profondeur des verbatims $^{[23,30,31]}$. Le travail prolongé sur le terrain, incluant, entre autres, une phase de planification avec les parties prenantes, assure une plus grande crédibilité ${ }^{[30]}$ de même que la prise en compte du contexte et de la réalité du milieu clinique. Des discussions au sein de l'équipe de recherche et une posture réflexive 
de la part de la chercheure, infirmière au service des urgences, ont facilité une meilleure compréhension du point de vue des personnes participantes ${ }^{[23,30]}$. Toutefois, en raison du lien de l'étudiante avec le milieu clinique, un biais de désirabilité sociale est possible. La triangulation des sources de données et des méthodes de collecte des données a par ailleurs contribué à la crédibilité des résultats.

La fiabilité consiste à vérifier la reproductibilité du déroulement de la recherche et des analyses ${ }^{[30]}$. La méthodologie décrite précédemment permet à d'autres chercheurs de porter un regard sur le processus de recherche et d'examiner la correspondance entre les résultats présentés et les stratégies de collecte et d'analyse des données utilisées.

La transférabilité (validité externe) renvoie au degré de similarité entre deux contextes et permet de transposer les résultats de la recherche à d'autres situations similaires ${ }^{[30]}$. Les besoins et l'activité de formation, décrits en profondeur dans le cadre de cette recherche, pourraient possiblement être similaires à ce qui est vécu dans d'autres centres de traumatologie secondaires du Québec mais la transférabilité à d'autres contextes demeure limitée.

Il a par ailleurs été impossible de contrôler certains facteurs entre les différents temps de mesure comme l'exposition à des populations de patients polytraumatisés ou encore la participation à d'autres activités de FC. Le questionnaire construit pour la recherche a fait l'objet d'une validation de contenu mais il y aurait lieu de procéder à une validation plus exigeante et de vérifier notamment sa validité de construit $^{[21,32]}$. Enfin, un dispositif de recherche incluant un groupe témoin permettrait une évaluation plus rigoureuse des effets de la FC.

L'élaboration d'une activité de FC « sur mesure », selon les besoins exprimés, constituait une nouveauté dans le domaine des soins infirmiers en traumatologie. Cette approche pourrait s'avérer prometteuse pour la réalisation de recherches futures. Les résultats font ressortir l'importance d'offrir des formations qui s'échelonnent dans le temps, qui se déroulent dans un contexte le plus réel possible et qui permettent aux infirmiers(ères) de développer certaines connaissances $^{[16]}$. D'autres recherches s'imposent pour documenter la conception d'activités de FC sur mesure ainsi que leurs effets sur la qualité des soins et leurs retombées auprès des patients.

\section{Contributions}

Mélanie Marceau a conçu le protocole de recherche. Elle a recueilli et analysé les données dans le cadre de sa maîtrise en sciences cliniques (sciences infirmières). En tant que directrices des travaux de recherche de Mélanie Marceau, Lise R. Talbot et Frances Gallagher ont contribué à l'ensemble des étapes du processus de recherche. Mélanie Marceau a écrit le manuscrit avec la collaboration de ses deux directrices.

\section{Déclaration d'intérêts}

Aucune auteure ne déclare de conflit d'intérêt relatif à l'objet de cet article.

\section{Approbation éthique}

L'étude a été approuvée par le Comité d'éthique de la recherche du centre hospitalier de Sherbrooke (décision : 06-054).

\section{Références}

1. Canadian Association of Critical Care Nurse. Standards for Critical Care Nursing Practice ( 3 e édition). London: CACCN, 2004.

2. Bahn D. Reasons for post registration learning: impact of the learning experience. Nurse Educ Today 2007;27:715-22.

3. Gallagher L. Continuing education in nursing: a concept analysis. Nurse Educ Today 2007;27:466-73.

4. Griscti O, Jacono J. Effectiveness of continuing education programs in nursing: literature review. J Adv Nurs 2006;55:449-56.

5. Flores Peńa Y, Alonso Castillo M. Factors influencing nursing staff member's participation in continuing education. Rev Lat Enfermagem 2006;14:309-15. 
6. Baird C, Kernohan G, Coates V. Outcomes of advanced trauma life support training: questioning the role of observer. Accid Emerg Nurs 2004;12:131-5.

7. Cole E. Assessment and management of trauma patient. Nurs Stand 2004;18:45-54.

8. Tippett J. Nurses' acquisition and retention of knowledge after trauma training. Accid Emerg Nurs 2004;12:39-46.

9. Chamberland G, Lavoie L, Marquis D. 20 Formules pédagogiques. Québec : Presses de l'Université du Québec, 1996.

10. Lasnier F. Réussir la formation par compétences. Montréal : Éditions Guérin, 2000.

11. Razongles P. Grands cadres de la formation de groupe. In : Gallois P(Ed.). La formation médicale continue : principes, organisations, objectifs, méthodes et évaluation. Paris : Flammarion Médecine-Sciences, 1997 : 184-9.

12. Robert E, Clément N. La formation continue : mode d'emploi. Paris : Éditions Lamarre, 1989.

13. Thomson O'Brien MA, Freemantle N, Oxman AD, Wolf F, Davis DA, Herrin J. Continuing education meetings and workshops: effects on professional practice and health care outcomes. J Contin Educ Health Prof 2001;3:187-8.

14. Curtis K. Nurse's experiences of working with trauma patients. Nurs Stand 2001;16:33-8.

15. Melnick DE. Physician performance and assessment and their effect on continuing medical education and continuing professional development. J Contin Educ Health Prof 2004;24:S38-S49.

16. Le Boterf G. Ingénierie et évaluation des compétences (5 édition). Paris : Éditions d'Organisation, 2006.

17. Tardif J. L'évaluation des compétences : documenter le parcours de développement. Montréal : Chenelière Éducation, 2006.

18. Vienneau R. Apprentissage et enseignement : théories et pratiques ( $2^{\mathrm{e}}$ édition). Montréal : Gaëtan Morin, 2011.

19. Guba EG, Lincoln YS. Fourth generation evaluation. Newbery Park: Sage Publications, 1989.

20. Emergency Nurse Association (ENA). Trauma Nursing Core Course: Provider Manuel (6th edition). Chicago: Emergency Nursing Association, 2007.
21. Burns N, Grove SK. The practice of nursing research: Appraisal, synthesis, and generation of evidence (6th edition). St-Louis, MO: Elservier Saunders, 2009.

22. Morgan DL, Kruger AK. The Focus Group Kit. Thousand Oaks: Sage Publications, 1998.

23. Huberman AM, Miles MB. Analyse de données qualitatives ( 2 édition). Bruxelles : De Boeck, 2003.

24. Armour KM, Yelling M. Effective professional development for physical education teachers: the role of informal, collaborative learning. J Teach Phys Educ 2007;26:177-200.

25. McKeachie W, Svinicki M. McKeachie's teaching tips: strategies, research, and theory for college and university teachers (13th edition). Belmont: Wadsworth Cengage Learning, 2011.

26. Huba ME, Freed JE. Learner-centered assessment on college campuses: shifting the focus from teaching to learning. Boston: Allyn and Bacon, 2000.

27. Hugues E. Nurses' perceptions of continuing professional development. Nurs Stand 2005;19:41-9.

28. Razongles P. Grands cadres de la formation de groupe. In : Gallois P (ed.). La formation médicale continue : principes, organisations, objectifs, méthodes et évaluation. Paris : Flammarion Médecine-Sciences, 1997 : 184-9.

29. Robert E, Clément N. La formation continue : mode d'emploi. Paris : Éditions Lamarre, 1989.

30. Laperrière A. Les critères de scientificité des méthodes qualitatives. In : Poupart J, Deslauriers JP, Groulx LH, Laperrière A, Mayer R, Pirès AP (Eds.). La recherche qualitative. Enjeux épistémologiques et méthodologiques. Montréal : Gaëtan Morin, 1997: 365-90.

31. Creswell JW. Qualitative inquiry \& research design. Choosing among five approaches (2nd edition). Thousand Oaks: Sage Publications, 2007.

32. Fortin MF. Fondements et étapes du processus de recherche: méthodes quantitatives et qualitatives (2e édition). Montréal : Chenelière Éducation, 2010.

Correspondance et offprints : Mélanie Marceau, École des sciences infirmières, Faculté de médecine et des sciences de la santé, Université de Sherbrooke; $300112^{\mathrm{e}}$ Avenue Nord, Sherbrooke, Québec, Canada, J1H 5N4.

Mailto : melanie.marceau@usherbrooke.ca 